\title{
Evaluation of entrance surface dose and scattered dose to the pelvis for common radiological examinations in analog and digital radiography: $A$ phantom study
}

\author{
A. Peiro' ${ }^{1}$, A. Danyaei ${ }^{1}$, N. Chegeni ${ }^{*}$, M. Tahmasbi ${ }^{2}$, J. Fatahiasl ${ }^{2}$ \\ ${ }^{1}$ Department of Medical Physics, School of Medicine, Ahvaz Jundishapur University of Medical Sciences, Ahvaz, Iran \\ ${ }^{2}$ Department of Radiology Technology, School of Allied Medical Sciences, Ahvaz Jundishapur University of Medical \\ Sciences, Ahvaz, Iran
}

\section{- Original article}

\section{*Corresponding author: Nahid Chegeni, Ph.D., E-mail: chegenin@gmail.com}

Revised: August 2020

Accepted: November 2020

Int. J. Radiat. Res., October 2021; 19(4): 937-945

DOI: $10.29242 /$ ijrr.19.4.937

\begin{abstract}
Background: Development of digital radiography revolutionized the field of medical imaging and increased the diagnostic accuracy. Despite its advantages, such as wide dynamic range and post-processing capacity, patient dosage has increased. The present study aimed to evaluate the entrance surface doses (ESDs) of primary beams to organs and the scatter dose received by pelvis in digital and analog systems with an emphasis on the radiation field size. Materials and Methods: A whole body phantom PBU-50, and TLD GR-200 chips, were used to measure ESDs. Radiation techniques used in analog and digital systems were implemented, using a Pars Pad radiography unit. Exposure factors in digital radiography were applied for both standard and clinically used radiation field sizes. Radiography was performed in the extremities, skull, chest, abdomen, pelvis, and lumbar spine. In each view, three dosimeters were placed on the phantom body to measure primary dose. Three others were also placed on pubic symphysis to measure scattered dose. Results: In digital and analog techniques, the difference in primary doses was significant for limbs, unlike large organs $(P=0.00)$. However, scattered dose to the pelvis was not significantly different $(\mathrm{P}=0.7417)$. Comparison of standard digital and clinically used field sizes showed significant differences in the scattered dose received by pelvis $(P=0.014)$, while the primary dose differences were not significant $(P=0.468)$. Conclusion: Inadequate radiation protection, especially the use of an improper radiation field size and misuse of digital system capabilities, can result in increased patient dosage.
\end{abstract}

Keywords: Analog radiography, digital radiography, ESDs, field size, radiation protection.

\section{INTRODUCTION}

Discovery of X-rays in 1895 by Wilhelm Conrad Rontgen opened new horizons in medical sciences. However, the challenging nature of ionizing radiation has highlighted the importance of protective measures. The highly penetrating nature of X-rays and differences in the sensitivity of body tissues to this type of radiation are responsible for the biological effects in humans. To limit the harmful tissue reactions and the stochastic effects of X-rays, the International Commission on Radiological Protection (ICRP) has recommended two principles of justification and optimization (1). Since radiological imaging has become one of the main diagnostic methods to determine the cause of diseases, some concerns have been raised regarding full adherence to protective and patient dose principles ${ }^{(2)}$. Accordingly, the ICRP 
introduced a diagnostic reference level (DRLs) to optimize the radiation protection of patients in 1996.

According to this standard, if the patient dose is unusually high or low in a particular procedure, a proper review is necessary. In such cases, the entrance surface dose (ESD) is measured by methods, such as thermoluminescence dosimetry (TLD) $(3,4)$. Imaging systems have substantially improved since the discovery of X-ray. Analog systems have been used for many years, and digital systems were developed in the 1980's $(5,6)$. The technology used in these well-accepted and widely used systems is based on computer processing. Advantages, such as lack of need for a dark room and chemical processing, image storage, wide dynamic ranges, and post-processing capabilities, have increased the speed and quality of imaging (7-9). However, these systems can increase the patient dosage due to different factors (10).

In 2004, the ICRP Publication 93 provided the necessary background information and emphasized on patient dose management in digital techniques (11). Unlike analog techniques, where low or high exposure results in a bright or dark image with no diagnostic information, digital systems may increase the patient dose without image disruption due to their wide dynamic range (11-13). Although some studies have reported dose reduction in these systems (14), some have shown an unnecessary increase in the average ESD with the development of digital radiography (15). Post-processing capabilities of digital systems, including the use of different filters and change of density, have reduced the need to consider proper radiation factors (16).

In digital systems, detectors are sensitive to noise; therefore, operators often tend to apply greater currents (mAs) to raise the signal-tonoise ratio $(\mathrm{S} / \mathrm{N})$, which in turn increases the patient dose (17). Also, radiation field size, which is an essential factor in scattered radiation, image quality, and patient dose, should be limited to the target area (18). According to some studies, due to many factors, such as high workload or convenience, field size localization is not properly adjusted $(19,20)$. On the other hand, electronic collimation in digital systems allows operators to use this capability rather than adjust the field size manually; therefore, the patient dose may increase by applying field sizes greater than needed $(21,22)$. There is a direct association between the stochastic effects and radiation doses below 100 millisieverts (mSv). The high risk and negative effects of cancer are expected at doses up to $100 \mathrm{mSv}$ (as a single or a cumulative dose over a year) (1).

Recent studies have shown that the radiographer's knowledge of radiation hazards is sometimes inadequate, and evaluation of protection standards is necessary $(23,24)$. The present study aimed to evaluate the ESDs of different organs in conventional radiography examinations using analog and digital radiography systems. Although many studies have compared radiography techniques in digital and analog systems, they have often investigated ESDs for organs with a greater thickness, such as the lumbar spine, pelvis, abdomen, skull, and chest $(15,25-27)$. In this study, we investigated ESDs in the extremities, as well. The scattered dose to the pelvis in these two imaging systems was also measured and compared. Moreover, the effect of field size collimation in digital techniques on the received dose of the pelvis due to examinations was evaluated.

This is the novelty of this study, as we used a radiography-specific whole-body phantom and adjusted the field size and radiography exposure factors according to the average values in the clinical setting of our teaching hospitals.

\section{MATERIALS AND METHODS}

\section{Imaging system and phantom}

Pars Pad X-ray machine (model PMX-600, Iran) was used for radiological examinations. The system was calibrated, and the accuracy of its performance was confirmed with a dose-area product (DAP) meter (model NE Technology, UK) for various kVps (Kilovoltage peak). An anthropomorphic whole-body phantom (model PBU-50, Japan), resembling the body mass

Int. J. Radiat. Res., Vol. 19 No. 4, October 2021 
(height of $1.65 \mathrm{~m}$ and weight of about $50 \mathrm{~kg}$ ), was scanned based on the study objectives, according to the scan protocols in table 1 .

\section{Exposure factors and scan protocols}

The average values of exposure factors in the analog and the digital techniques were determined, using a questionnaire by technicians in different centers (20). In digital technique, the average values of automatic exposure factors in different examinations were measured by a flat panel detector (model Davinci premium, Italy). Since we focused on the exposure factors and field sizes of digital and analog imaging systems, the same radiography unit was utilized, which eliminated the effects of inherent and added filtration and backscattering of the examination table (patient table) and also kept environmental factors, such as temperature and humidity, constant. The ESDs were also determined for standard and clinically used field sizes in the digital technique.

The standard field size in different examinations was determined according to Merrill's Atlas of Radiographic Positioning \& Procedures, which is known as a guideline for diagnostic radiology techniques (18). Also, the clinically used field sizes (non-standard) were obtained according to the reference (20) and then adjusted to the anthropomorphic phantom dimensions (table 1).

Table 1. Scan protocols in analog and digital techniques (completed digital techniques with standard and clinically used field

\begin{tabular}{|c|c|c|c|c|c|c|c|c|c|c|c|}
\hline \multirow{3}{*}{ Organs } & & \multicolumn{3}{|c|}{$k V p$} & \multicolumn{3}{|c|}{ mAs } & \multirow{2}{*}{\multicolumn{2}{|c|}{$\begin{array}{c}\text { Focal Film Distance }(\mathrm{cm}) \\
\text { Digital }\end{array}$}} & \multirow{2}{*}{\multicolumn{2}{|c|}{$\begin{array}{c}\text { Field Size }\left(\mathrm{cm}^{2}\right) \\
\text { Digital }\end{array}$}} \\
\hline & & \multicolumn{2}{|c|}{ Digital } & \multirow[b]{2}{*}{ Analog } & \multicolumn{2}{|c|}{ Digital } & \multirow[b]{2}{*}{ Analog } & & & & \\
\hline & & standard & $\begin{array}{c}\text { clinically } \\
\text { used }\end{array}$ & & standard & $\begin{array}{c}\text { clinically } \\
\text { used }\end{array}$ & & standard & $\begin{array}{c}\text { clinically } \\
\text { used }\end{array}$ & Standard $^{(18)}$ & $\begin{array}{l}\text { clinically }^{(20)} \\
\text { used }^{(20}\end{array}$ \\
\hline \multirow{2}{*}{ Skull } & AP & 65 & 65 & 63 & 25 & 25 & 25 & 100 & 100 & $20 \times 32$ & $30 \times 35$ \\
\hline & LA & 60 & 60 & 57 & 20 & 20 & 12 & 100 & 100 & $28 \times 32$ & $42 \times 35$ \\
\hline Chest & $A P$ & 70 & 70 & 58 & 10 & 12 & 30 & 100 & 110 & $40 \times 35$ & $54 \times 45$ \\
\hline Abdomen & $A P$ & 72 & 72 & 60 & 30 & 40 & 25 & 100 & 110 & $35 \times 43$ & $54 \times 45$ \\
\hline Pelvis & AP & 68 & 68 & 57 & 30 & 40 & 40 & 100 & 110 & $43 \times 35$ & $45 \times 45$ \\
\hline Arm & AP\&LA & 58 & 58 & 49 & 15 & 15 & 4 & 100 & 100 & $18 \times 43$ & $28 \times 50$ \\
\hline Forearm & AP\&LA & 55 & 55 & 44 & 8 & 8 & 4 & 100 & 100 & $13 \times 38$ & $22 \times 50$ \\
\hline Femur & AP\&LA & 70 & 74 & 58 & 20 & 20 & 40 & 100 & 110 & $20 \times 43$ & $32 \times 54$ \\
\hline Leg & AP\&LA & 60 & 64 & 49 & 12 & 12 & 4 & 100 & 110 & $15 \times 43$ & $26 \times 54$ \\
\hline \multirow{2}{*}{ Lumbar } & $A P$ & 70 & 70 & 68 & 40 & 40 & 25 & 100 & 110 & $23 \times 35$ & $25 \times 54$ \\
\hline & LA & 85 & 85 & 77 & 40 & 40 & 50 & 100 & 110 & $20 \times 35$ & $25 \times 54$ \\
\hline
\end{tabular}

\section{Scanned organs}

Radiography of the arms, forearms, legs, femurs, skull, and lumbar spine was performed in AP and LA views, and the chest, abdomen, and pelvis were scanned in the AP view. The scanned organs and scan protocols for the analog and digital techniques are shown in table 1; the digital technique was performed with standard and clinically used field sizes.

\section{Dosimetry}

Thermoluminescence dosimeter chips (TLD GR-200 series LiF: $\mathrm{Mg}, \mathrm{Cu}, \mathrm{P}$, China )with a diameter of $4.5 \mathrm{~mm}$ and a thickness of $0.8 \mathrm{~mm}$ were used to measure ESDs. These chips do not perturb radiation field due to their small sizes and with the atomic number similar to that of soft tissue. First, the chips were annealed in an electric furnace (model EXCITON, Iran) at $240^{\circ} \mathrm{C}$ for 11 minutes according to the reference (28). To compensate the variations in TLDs response, ECC (element correction coefficient) factor for individual TLDs were obtained.

It is necessary to irradiate all TLD chips in the sample, with the same dose. Therefore; all TLDs were exposed to $100 \mathrm{cGy}$ dose of $6 \mathrm{MV}$ photons (Siemens Primus plus LINAC) with a $1.5 \mathrm{~cm}$ Plexiglas slab as the buildup cap. Reading of all 
chips were averaged and divided to reading of anyone. To determine calibration curve, TLD chips were divided into 20 groups of 5 and then exposed to different doses of X-ray tube within diagnostic radiology range (from 1.23 to 19.183 mGy) $(28,29)$. According to the energy range used in different examinations (between 44 to $85 \mathrm{kVp}$ mentioned in table 1 ), we used $80 \mathrm{kVp}$ to plot the calibration curve.

The output of the tube was measured in TLD location by Solidose RTI detector (from Sweden) with a maximum error of 3\%. TLD Reader (Harshaw 3500, USA), was used to read the chips. The reading of each TLD was multiplied by corresponding ECC factor and the average value for each group was calculated. Then calibration curve was plotted against dose (figure 1) and the relationship between absorbed dose and TLD response was calculated by fitting the data (equation 1 ):

$$
\mathrm{Y}(\mathrm{nC})=69.4 \times \mathrm{X}(\mathrm{mGy})-55.8
$$

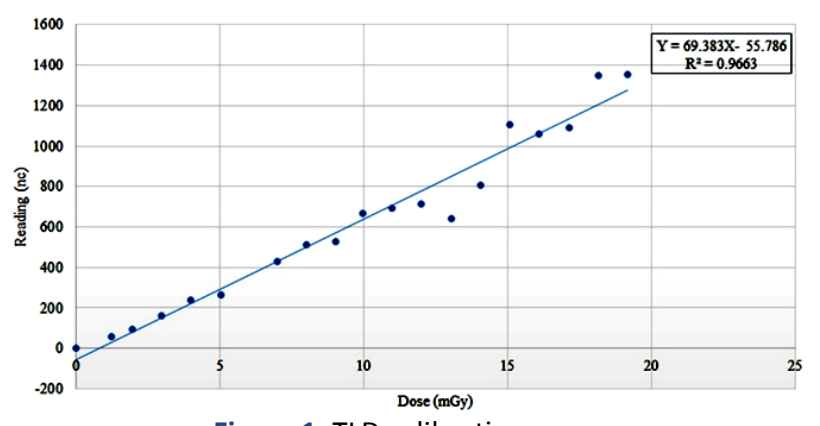

Figure 1. TLD calibration curve.

Where; $Y$ represents TLD readings in nanocolumb (nC) and $X$ represents dose in miligray (mGy).

In any examination phase, a bag of three TLD chips were placed to measure environmental background radiation dose. Read out process were performed within $0-24 \mathrm{~h}$ after exposure and values were obtained in nano Coulomb (nC). Next all three chips in each projection view were corrected by ECC factor and averaged then corrected for background reading. Finally, reading of all chips converted to dose in Milligray (mGy) by the calibration curve equation.

In each radiographic view, a group of three
TLD chips covered in radiolucent bag, were placed at central axis of radiation field on the phantom body, to measure the primary dose. Three other TLDs were placed on the pubic symphysis to measure the scattered dose. A fixed group of TLDs was placed on the pubic symphysis to measure the scattered radiation dose arising from extremity examinations (arm, forearm and leg, AP \& LA view and right \& left side) and the same work was done for skull (AP \& LA view) (table 1). The reason was that the production of scatter in the imaging of the extremities was less due to their low thickness. Likewise, because the skull is located at a distance from the pelvis, the scattered dose production per view was small.

\section{Mathematical calculations and statistical analysis}

Calculations were performed in Microsoft office Excel 2016, and data were analyzed using SPSS (Armonk, NY, USA: IBM Corp). Kolmogorov-Smirnov test was used to examine the normal distribution of data. Based on the results, the collected data were not normally distributed; therefore, non-parametric Mann-Whitney U test was used for data analysis. P-values $<0.05$ were considered statistically significant.

\section{RESULTS}

The mean ESDs are presented in table 2 . Measurements were performed for standard field sizes in the analog technique and for standard and clinically used field sizes in the digital technique. Table 2 shows the ESDs from the primary beam for each studied organ. Considering the similarity of radiation parameters and the received doses of the limbs, as summarized in the table, the primary ESDs for the arms, forearms, legs, and femurs of both sides in both projections (AP-LA) were averaged per view. Also, the scattered doses received by the pubic symphysis due to different examinations are shown in table 2. Figure 2 presents the comparison of scattered doses received by the pubic symphysis due to different 
examinations for standard and clinically used field sizes in the digital system. Values are expressed in mGy and averaged per view for organs with different sides (right and left) and projections (AP-LA).

All radiological examinations were performed using the digital technique in both standard and clinically used radiation field sizes. Table 3 presents the statistical comparison of primary and scattered doses in the analog and digital techniques. For more accuracy, because of differences in the radiation parameters of the limbs and large organs (table 1), two separate rows were considered for the primary ESDs. Table 4 shows the measured ESDs versus the diagnostic reference levels (DRLs) reported in some previous studies. The recorded values (in mGy) are related to the digital technique with standard field sizes (30-34).

Table 2. The measured primary and secondary mean entrance surface doses for the studied radiologic examinations.

\begin{tabular}{|c|c|c|c|c|c|c|c|}
\hline \multirow{3}{*}{ Organs } & & \multicolumn{3}{|c|}{ Primary dose (mGy) } & \multicolumn{3}{|c|}{ Scatter dose to pelvis (mGy) } \\
\hline & & \multicolumn{2}{|c|}{ Digital } & \multirow{2}{*}{ Analog } & \multicolumn{2}{|c|}{ Digital } & \multirow{2}{*}{ Analog } \\
\hline & & standard & clinically used & & standard & clinically used & \\
\hline \multirow{2}{*}{ Skull } & AP & 1.72 & 1.66 & 1.63 & \multirow{2}{*}{$0.81 \pm 0.00^{\dagger}$} & \multirow{2}{*}{$0.82 \pm 0.00^{\dagger}$} & \multirow{2}{*}{$0.80 \pm 0.00^{\dagger}$} \\
\hline & LA & 1.39 & 1.40 & 1.07 & & & \\
\hline Chest & AP & 1.24 & 1.30 & 1.72 & $0.81 \pm 0.00$ & $0.83 \pm 0.00$ & $0.81 \pm 0.00$ \\
\hline Abdomen & AP & 2.27 & 2.34 & 1.60 & $0.90 \pm 0.00$ & $2.14 \pm 0.00$ & $0.87 \pm 0.00$ \\
\hline Pelvis & AP & 2.12 & 2.03 & 1.95 & - & - & - \\
\hline Arm & AP\&LA & $1.15 \pm 0.02$ & $1.12 \pm 0.02$ & $0.85 \pm 0.00$ & \multirow{3}{*}{$0.81 \pm 0.00^{\ddagger}$} & \multirow{3}{*}{$0.99 \pm 0.00^{\ddagger}$} & \multirow{3}{*}{$0.81 \pm 0.00^{\ddagger}$} \\
\hline Forearm & AP\&LA & $0.95 \pm 0.01$ & $0.92 \pm 0.02$ & $0.84 \pm 0.01$ & & & \\
\hline Leg & AP\&LA & $1.08 \pm 0.06$ & $1.01 \pm 0.03$ & $0.85 \pm 0.01$ & & & \\
\hline Femur & AP\&LA & $1.56 \pm 0.05^{*}$ & $1.37 \pm 0.06^{*}$ & $1.75 \pm 0.02^{*}$ & $0.84 \pm 0.02^{*}$ & $1.11 \pm 0.04^{*}$ & $0.84 \pm 0.01^{*}$ \\
\hline \multirow{2}{*}{ Lumbar } & AP & 2.55 & 2.33 & 1.81 & $0.85 \pm 0.00$ & $1.32 \pm 0.00$ & $0.82 \pm 0.00$ \\
\hline & LA & 3.82 & 3.40 & 3.34 & $0.85 \pm 0.00$ & $1.99 \pm 0.00$ & $0.82 \pm 0.00$ \\
\hline
\end{tabular}

* average scatter of arm, forearm and leg, right and
$*$ average of right and left side and AP-LAT position

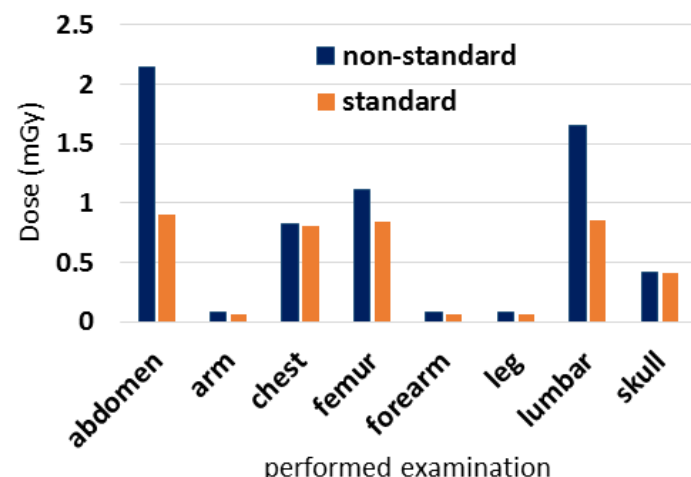

Figure 2. Scattered doses (secondary ESDs) received by symphysis pubis, related to the studied radiographies performed by digital technique with standard and clinically used (non-standard) field sizes.
Table 3. Comparison of primary and scattered doses in analog and digital techniques (standard vs. clinically used field sizes for digital technique).

\begin{tabular}{|c|c|c|c|c|}
\hline \multicolumn{5}{|c|}{ Radiology System: Digital vs. Analog } \\
\hline Dose (mGy) & Radiology System & $\mathbf{N}$ & Mean \pm SD & p-value \\
\hline \multirow{2}{*}{$\begin{array}{l}\text { Primary dose of } \\
\text { massive organs }^{1}\end{array}$} & Digital & 11 & $1.94 \pm 0.74$ & \multirow{2}{*}{0.554} \\
\hline & Analog & 11 & $1.83 \pm 0.55$ & \\
\hline \multirow{2}{*}{$\begin{array}{c}\text { Primary dose to } \\
\text { extrimities }^{2}\end{array}$} & Digital & 12 & $1.1 \pm 0.09$ & \multirow{2}{*}{$0.00^{*}$} \\
\hline & Analog & 12 & $0.85 \pm 0.00$ & \\
\hline \multirow{2}{*}{$\begin{array}{c}\text { scatter dose received } \\
\text { to pelvis from all } \\
\text { examinations }\end{array}$} & Digital & 22 & $0.76 \pm 0.18$ & \multirow[b]{2}{*}{0.7417} \\
\hline & Analog & 22 & $0.76 \pm 0.18$ & \\
\hline \multicolumn{5}{|c|}{ Digital: Standard vs. clinically used filed sizes } \\
\hline \multirow{2}{*}{$\begin{array}{c}\text { Primary Dose to } \\
\text { Organs }\end{array}$} & standard field size & 23 & $1.48 \pm 0.67$ & \multirow{2}{*}{0.468} \\
\hline & clinically used size & 23 & $1.39 \pm 0.60$ & \\
\hline \multirow{2}{*}{$\begin{array}{c}\text { Scatter Dose to } \\
\text { Pelvis }\end{array}$} & standard field size & 22 & $0.76 \pm 0.18$ & \multirow{2}{*}{$0.014^{*}$} \\
\hline & clinically used size & 22 & $1.14 \pm 0.54$ & \\
\hline \multicolumn{5}{|c|}{$\begin{array}{l}\text { 1. Skull, chest, abdomen, pelvis, lumbar, femur } \\
\text { 2. Arm, forearm, leg } \\
\text { * means the difference is significant. }\end{array}$} \\
\hline
\end{tabular}


Table 4. The comparison of the measured mean entrance surface dose (ESD) values with diagnostic reference level (DRLs) of some studies.

\begin{tabular}{|c|c|c|c|c|c|c|}
\hline \multirow{2}{*}{ Organ } & \multicolumn{2}{|c|}{$\begin{array}{c}\text { ESD } \\
\text { (mGy) }\end{array}$} & \multicolumn{5}{|c|}{ Diagnostic Reference Level (DRLs) } \\
\cline { 2 - 7 } & $\begin{array}{c}\text { This } \\
\text { study }\end{array}$ & IAEA $^{(30)}$ & UK $^{(31)}$ & JAPAN $^{(32)}$ & IRAN $^{(33)}$ & IRAN $^{(34)}$ \\
\hline Skull (AP) & 1.72 & 2.41 & 1.8 & 3 & 1.22 & 1.3 \\
\hline Skull (LA) & 1.39 & - & 1.1 & 2 & 1.01 & 1.17 \\
\hline $\begin{array}{c}\text { Chest } \\
\text { (AP) }\end{array}$ & 1.24 & - & 0.2 & - & 0.64 & - \\
\hline $\begin{array}{c}\text { Abdomen } \\
\text { (AP) }\end{array}$ & 2.27 & 3.64 & 4.4 & 3 & 2.15 & 2 \\
\hline $\begin{array}{c}\text { Pelvis } \\
\text { (AP) }\end{array}$ & 2.12 & 3.68 & 3.9 & 3 & 1.47 & 1.62 \\
\hline $\begin{array}{c}\text { Lumbar } \\
\text { (AP) }\end{array}$ & 2.55 & 4.07 & 5.7 & 4 & 1.99 & 2.69 \\
\hline $\begin{array}{c}\text { Lumbar } \\
\text { (LA) }\end{array}$ & 3.82 & 8.53 & 10 & 11 & 3.83 & 4.22 \\
\hline
\end{tabular}

\section{DISCUSSION}

In this study, we aimed to evaluate the ESDs of different organs for analog and digital radiography systems. We focused on the effects of radiation field size on the scattered doses received by the pelvic cavity in different examinations. Major attention must be paid to the increased use of digital radiography systems because of their various options and simple operation. The unique features of these systems, such as a wide dynamic range and post-processing capacity, may lead to patient overexposure.

Our results revealed a significant decrease in the primary doses of the extremities (i.e., arms, forearms and legs), using the analog radiography system ( $p$-value $=0.00$ ) (table 3 ). On the other hand, the mean ESDs of large organs (i.e., skull, chest, abdomen, lumbar spine, and femur) were almost similar and independent of the radiography system type ( $\mathrm{p}$-value $=0.554$ ) (table 3). On the contrary, in a study by Seo et al. (15), the average ESD in digital radiography was $55.25 \%$ higher than that of analog radiography. However, there was no significant difference in the primary ESDs of large organs with the two systems. Nevertheless, because of the wide dynamic range, digital systems have the potential to increase the radiation parameters and patient dose without any disruption in the image quality (11).

Some studies have evaluated this issue while emphasizing the importance of guidelines for conventional radiography. Overall, dose control and balance between image quality and patient dose should be considered in digital systems (12, 16). Moreover, the signal-to-noise ratio $(\mathrm{S} / \mathrm{N})$, in digital systems has become a serious issue. Operators face limitations in reducing $m A s$ and tend to apply higher radiation exposures to improve the image quality (17). Also, anti-scatter grids are used for almost all examinations in digital systems, which require higher radiation parameters to compensate for the effect of grid (35). On the other hand, in radiography of the limbs with analog systems, anti-scatter grids are not used.

Based on the present findings (table 1), the average $\mathrm{kVp}$ was about $20 \%$ higher in digital techniques, and the average mAs was three times greater in imaging of the extremities. Recently, Mohsenzadeh et al. (36) evaluated the patient dose in routine digital radiography. They found considerable variations in ESDs between different imaging centers; differences were even observed at the same center using the same digital systems. Their findings indicated many influential factors, such as patient setup, exposure setting (kVp, mAs, field size), use of grids, and skillfulness of the radiology staff. Although digital devices can remove the grid, radiographers are often reluctant to separate it due to its sensitivity. However, ESD or even ED (effective dose) may seem insignificant in the extremities, all safety precautions must be observed for radiation protection.

In addition, according to the results of the present study, the average scattered dose received by the pubic symphysis showed no significant difference in the analog and digital techniques ( $\mathrm{p}$-value=0.7417) (table 3 ), which can be attributed to the similar performance of these two systems for large organs. Also, in imaging of the extremities, the low energy of primary beams, which leads to less scattered radiation, may be influential. Moreover, an appropriate field size is a major factor in

Int. J. Radiat. Res., Vol. 19 No. 4, October 2021 
radiation protection that may be ignored.

A recent study by Farzanegan et al. (20) reported proper field size collimation in only $46 \%$ of examinations. Some factors may be involved in the low rate of field size collimation, such as patients' emergency conditions, high staff workload, concerns about missing the diagnostic targets, and technician's neglect $(19,20$, 36). Some specific capabilities of digital radiography systems, such as electronic collimation of field size, have caused some challenges. In this regard, Zetterberg et al. (22) reported an increase in the irradiated field size since the implementation of digital radiography, which caused technicians to neglect the alignment of radiation field size manually. In analog techniques, an increase in field size leads to foggy images, while image density and contrast can be moderated by post-processing filters in digital systems. Therefore, due to the increased use of digital radiology (36), field size inaccuracy seems to be more common in these systems. Accordingly, we designed an examination for digital systems with standard and clinically used field sizes.

The results revealed significant differences in the scattered dose of the pelvis for standard and clinically used field sizes in the digital technique ( $p$-value $=0.014$ ), while the primary ESDs were almost similar for both standard and clinically used radiation field sizes ( $\mathrm{p}$-value $=0.468$ ) (table 3), because the TLDs were located at the central beam axis, and exposure factors were the same in both field size plans. On the other hand, the patient dose increased in clinically used field sizes, where wider regions were irradiated, and the fields overlapped. This finding is consistent with the results reported by Fauber et al. (21), which showed a 27-60\% decrement in abdominal exposure during lumbar spine imaging by field size collimation. Also, as a wider region was exposed, the scattered photons were produced in a greater volume ${ }^{(35)}$.

According to the present findings (table 3), significant differences were found in the scattered doses received by the pubic symphysis for collimated and non-collimated field sizes as expected. We observed that part of doses received by TLDs in the pelvic cavity originated from the primary field borders of other examinations, where the primary beam reached the pubic symphysis, especially in noncollimated field sizes. As expected, abdominal, lumbar, and femur examinations had greater contributions to scatter radiation, which was particularly more pronounced in non-restricted field sizes. According to these findings, imaging of these organs should be done with more caution due to their proximity to the gonads; obviously, the condition deteriorates for obese patients and more importantly for children. In a study by Mohsenzadeh et al. (36), the same exposure parameters were implemented in some centers for both children aged 10-15 years and adults.

Our results are consistent with the findings reported by Ahmed et al. (37), which showed that when the radiation field opened at maximum size, scattered radiation received by the gonads was significantly higher. In abdominal examinations with improper adjustment of field size, the gonadal dose was 17 times higher. Since radiography is an important part of diagnostic procedures, appropriate optimization is necessary in every step of this procedure. Accordingly, a DRL has been introduced to maintain proper radiation exposure and avoid an unnecessary increase in the patient dose. Based on the present findings (table 4), the measured ESDs were less than DRLs reported by the International Atomic Energy Agency (IAEA) project and Japan $(30,32)$. These values were higher than the national DRLs (NDRLs) and local DRLs (LDRLs), reported in two Iranian studies in the lumbar LAT view $(33,34)$.

In comparison with the NDRL reported in the UK, the mean ESDs of the present study, except for the skull (LAT view) and chest (AP view), were lower (31). This study has some limitations, such as the use of a fixed-size phantom. We suggest further studies with phantoms of different sizes and thicknesses or collection of data from patients to compare the scattered radiation for different field sizes and body masses. 


\section{CONCLUSION}

Substitution of analog radiography systems with digital ones has highlighted the importance of specific measures and considerations. Although proper radiation conditions must be established in both systems, in digital units, justification and optimization are needed. According to the present findings, despite the limitations of signal-to-noise ratio, if the diagnostic imaging quality is maintained, operators can apply lower radiation factors in radiography of the extremities. Also, in some examinations, the received dose to the pelvis may exceed the recommended reference level, especially in non-restricted field sizes, and needs to be controlled. The present study revealed that protective measures may be ignored for various reasons. This is an issue of major concern, and safety principles, especially for younger age groups, must be optimized.

\section{ACKNOWLEDGMENTS}

This study is a part of MSc thesis of Ameneh Peiro which financially supported by Jundishapur Ahvaz University of Medical Sciences (Grant No. U -98154). The authors would like to thank the Radiologic Technology Department of Allied Medical Sciences Faculty for their sincere cooperation.

\section{Conflicts of interest: Declared none.}

\section{REFERENCES}

1. Valentin J (2008) The 2007 recommendations of the international commission on radiological protection. Elsevier International Commission on Radiological Protection, 330 pages.

2. Nations $U$ (2011) Sources and effects of ionizing radiation: United Nations Scientific Committee on the Effects of Atomic Radiation: UNSCEAR 2008 Report to the General Assembly, with Scientific Annexes: United Nations; 2011.

3. ICRP Publication 73 (1996). Radiological protection and safety in medicine. Annals of the ICRP, 6(2).
4. Lemoigne Y, Caner A, Rahal G (2007) Physics for medical imaging applications: Springer Science \& Business Media.

5. Moore R (1980) Computed radiography. Medical Electronics, 11(2): 78-9.

6. Sonoda M, Takano M, Miyahara J, Kato H (1983) Computed radiography utilizing scanning laser stimulated luminescence. Radiology, 148(3): 833-8.

7. Körner M, Weber $\mathrm{CH}$, Wirth S, Pfeifer K-J, Reiser MF, Treitl $M$ (2007) Advances in digital radiography: Physical principles and system overview. Radio Graphics, 27(3): 675-86.

8. Persliden J, Beckman KW, Geijer H, Andersson T (2002) Dose-image optimisation in digital radiology with a direct digital detector: an example applied to pelvic examinations. European Radiology, 12(6):1584-8.

9. Reiner BI and Siegel EL (2002). Technologists' Productivity When Using PACS: Comparison of Film-Based Versus Filmless Radiography. American Journal of Roentgenology, 179 (1): 33-7.

10. Vano E, Fernández JM, Ten JI, Prieto C, Gonzalez L, Rodriguez $R$, et al. (2007) Transition from screen-film to digital radiography: evolution of patient radiation doses at projection radiography. Radiology, 243(2): 461-6.

11. Vano E (2005) ICRP recommendations on 'Managing patient dose in digital radiology'. Radiation Protection Dosimetry, 114(1-3): 126-30.

12. Willis CE (2004) Strategies for dose reduction in ordinary radiographic examinations using CR and DR. Pediatric Radiology, 34(3): S196-200.

13. Willis CE and Slovis TL (2004) The ALARA concept in pediatric $C R$ and $D R$ : dose reduction in pediatric radiographic exams--a white paper conference executive summary. Pediatric Radiology, 34(3): S162-4.

14. Strotzer M, Gmeinwieser J, Volk M, Frund R, Seitz J, Manke C, et al. (1998) Clinical application of a flat-panel X-ray detector based on amorphous silicon technology: image quality and potential for radiation dose reduction in skeletal radiography. AJR American Journal of Roentgenology, 171(1): 23-7.

15. Seo D, Jang S, Kim J, Kim J, Sung D, Kim H, et al. (2013) A comparative assessment of entrance surface doses in analogue and digital radiography during common radiographic examinations. Radiation Protection Dosimetry, 158(1): 22-7.

16. Uffmann M and Schaefer-Prokop C (2009) Digital radiography: the balance between image quality and required radiation dose. European Journal of Radiology, 72(2): 2028.

17. Doyle $P$ (2009) Assessment and optimisation of digital radiography systems for clinical use: University of Glasgow.

18. Long B, Rollins J, Smith B (2016) Merrill's atlas of radiographic positioning and radiological procedure. Pennsylvania, Mosby.

19. Okeji MC, Anakwue AM, Agwuna K (2010) Radiation exposure from diagnostic radiography: an assessment of X-ray beam collimation practice in some Nigerian Hospitals. Internet Journal of Medical Update-EJOURNAL, 5(2).

Int. J. Radiat. Res., Vol. 19 No. 4, October 2021 
20. Farzanegan Z, Tahmasbi M, Cheki M, Yousefvand F, Rajabi M (2020) Evaluating the principles of radiation protection in diagnostic radiologic examinations: collimation, exposure factors and use of protective equipment for the patients and their companions. Journal of Medical Radiation Sciences, 67(2): 119-27.

21. Fauber TL and Dempsey MC (2013) X-ray field Size and patient dosimetry. Radiologic Technology, 85(2): 155-61.

22. Zetterberg LG and Espeland A (2011) Lumbar spine radiography-poor collimation practices after implementation of digital technology. The British Journal of Radiology, 84 (1002): 566-9.

23. Abuzaid M, Elshami W, Shawki M, Salama D (2019) Assessment of compliance to radiation safety and protection at the radiology department. Int J Radiat Res, 17(3): 439-46.

24. Alhasan M, Abdelrahman M, Alewaidat H, Khader Y (2016) Radiation dose awareness of radiologic technologists in major Jordanian hospitals. Int J Radiat Res, 14(2): 133-8.

25. Zhang M and Chu C (2012) Optimization of the radiological protection of patients undergoing digital radiography. Journal of Digital Imaging, 25(1): 196-200.

26. Tamboul JY, Yousef M, Mokhtar K, Alfaki A, Sulieman A (2014) Assessment of entrance surface dose for the patients from common radiology examinations in Sudan. Life Science Journal, 11(2): 164-8.

27. Panicker T, Tina-Angelina J, Korath MK, Mohandas K, Jagadeesan K (2013) Entrance skin dose estimation in Xray lumbar spine lateral procedure: Conventional vs digital X-ray units: A pilot study. JIMSA, 26(4): 219-20.

28. Duggan L, Hood C, Warren-Forward $H$, Haque $M$, Kron $T$ (2004) Variations in dose response with X-ray energy of LiF: $\mathrm{Mg}, \mathrm{Cu}, \mathrm{P}$ thermoluminescence dosimeters: implications for clinical dosimetry. Physics in Medicine \& Biology, 49(17): 3831.

29. Valentin J (2001) Radiation and your patient: A guide for medical practitioners. Annals of the ICRP, 31: 1-52
30. Muhogora WE, Ahmed NA, Almosabihi A, Alsuwaidi JS, Beganovic A, Ciraj-Bjelac O, et al. (2008) Patient doses in radiographic examinations in 12 countries in Asia, Africa, and Eastern Europe: initial results from IAEA projects. American Journal of Roentgenology, 190(6): 1453-61.

31. Hart D, Hillier M, Shrimpton P (2010) Doses to patients from radiographic and fluoroscopic $\mathrm{X}$-ray imaging procedures in the UK. Chilton: Health Protection Agency Centre for Radiation, Chemical and Environmental Hazards.

32. Yonekura $Y$ (2019) Diagnostic reference levels based on latest surveys in Japan-Japan DRLs 2015. Japanese Network for Research and Information on Medical Exposure. Medical Exposure Research Information Network (J-RIME).

33. Zarghani H (2018) Local diagnostic reference levels for some common diagnostic X-Ray examinations in Sabzevar county of Iran. Iranian Journal of Medical Physics, 15(1): 62-5.

34. Mohsenzadeh B, Deevband MR, Pouriran R (2018) The national diagnostic reference level in routine digital radiography examinations in Iran. Biomedical Journal of Scientific \& Technical Research, 7(5): 6183-6192.

35. Bushong SC (2013) Radiologic science for technologists-Ebook: physics, biology, and protection: Elsevier Health Sciences.

36. Mohsenzadeh B, Deevband MR, Paydar R, Ghorbani M (2020) Assessment of patient dose in routine digital radiography in Iran. Int J Radiat Res, 18(3): 449-60.

37. Ahmed AA, Shaddad IA, editors (2003) Measurement of dose received by patients from scattered radiation in diagnostic radiology in Khartoum. Proceedings of the Sixth Arab Conference on the Peaceful Uses of Atomic Energy, Volll Scientific Presentation (Reactors, Materials, Fuel Cycles and Nuclear Safety); Egypt. 
